\title{
Research and Application of Plastic Art Materials in Interior Design
}

\author{
Zhe Wang ${ }^{1}$, Ximu Yan ${ }^{2}$, Li Sun ${ }^{3}$ \\ Qinhuangdao Institute of Technology, 066100 , Qinhuangdao, Hebei, China \\ 123515821@qq.com
}

Keywords: material, properties, using, interior design.

\begin{abstract}
Materials in the development of the whole human art design play a role, played by no doubt, penetrating art, life, society and science fields. Molding material is the material base of interior design, as the design of chemical engineering works, whether to use function, or the satisfaction of mental function, is realized through the selection and construction of materials, As interior design art, the research on the modeling material is focused on the exploration of the reproduction and the performance, and thus lead to the exploration of the artistic features of the modeling materials. In this article, mainly discusses the importance of materials used in interior design, the properties of materials is analyzed and the use method.
\end{abstract}

\section{Introduction}

An important phenomenon of the design activity is that every time the emergence of new materials, will lead to a new design movement, the formation of the driving force for the development of design culture. The so-called "clothes make the man, Buddha depends on gold", The performance of interior design cannot be separated from the application of materials. Material to the designer, such as the writer of the text, the artist's pen, paint, paper, the sound of the music, copper iron, stone, In short, the importance of understanding the material, understanding of the properties of the material, you can easily create a handy. The correct choice of materials, not only to show the designer's design concept, giving space to the soul, so that space to speak, but also to reflect the human care, to meet the aesthetic needs of modern people. In addition to a very good combination of various materials, complement each other, the details of the building, you can enrich the vision, and the lure and touch, hearing, smell, etc. create space characteristics.

\section{Material Properties}

Vision is a window to understand the world, the objective world of human visual organs, the formation of information through the visual organs, so that people feel and understand. World is through the sense and the material of touch being perceived theory, this theory suggests that the material and color of visual communication function and the mode of human perception of entity and space. In indoor environment, because people stay the longest, and all kinds of materials to produce the shortest distance, therefore, for the material texture, touch, color, texture should be enough attention.

Texture. The texture of the material mainly includes the concept of two different levels: one is the texture elements in the form of the "texture", namely geometry detail characteristics of material surface; the second is the texture elements "texture", namely material surface physicochemical category feature. Different textures of materials give people different visual infectious force and tactile feeling such as the material thickness, and warm, soft, smooth, dry and wet, patterns and other and its categories, mechanical properties, processing and so on. In the design to experience for people on the material of the visual and tactile feeling to be strengthening and abstract, to the emotional state of matter, transfer to the user. Like Tadao Ando, he of the cement, glass, metal, cold machine use, contains the zen. Modern industrial materials concrete in the hands of Ando recovered it is bold and unrestrained, instead of Japan simple and elegant, concise, plain and the aesthetic view of the pursuit and expression. As shown in Figure 1 


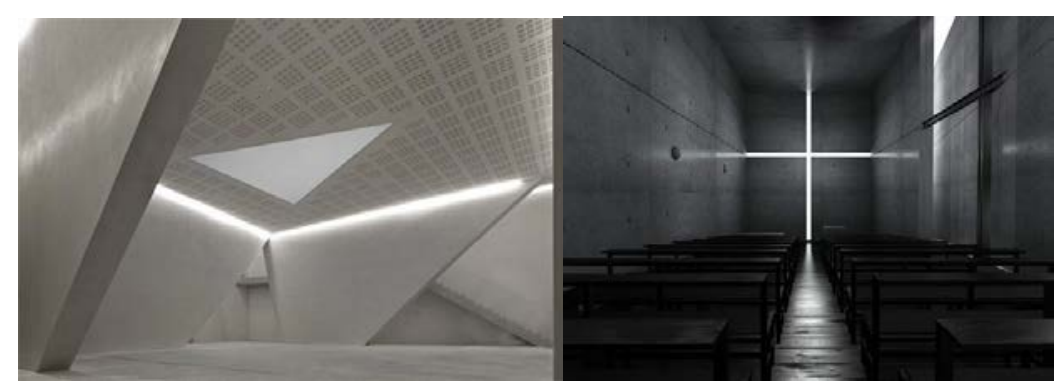

Fig 1. Tadao Ando- light and wind

Color. Color as the most sensitive, the most expressive visual elements, for the use of color in interior design very heavy. Color relative shape and texture, it produces the aesthetic feeling of tend to be more direct, synthetic-aperture power. Use of color can create an atmosphere of space, or elegant, warm fresh and romantic, beautiful and rich warm, excitement, celebrate joy, simplicity generous, use of color also divide the space, cause people in size, weight, temperature, expansion and contraction, forward, far and near etc. mental physical feeling. Solid color is natural color, in the interior design and construction without further processing, such as a variety of granite, marble, decorative panels, wall paper, fire board, plastic aluminum plate, etc. The colors of these materials should be reflected in the design of their own beauty, it is necessary for designers to choose and use according to the specific environment needs. Secondary color is according to the design of the space environment demand, changing color, in the use of materials for color processing; adjust the natural color of material, In order to achieve the harmony of indoor space color or contrast. Natural color and secondary color as shown in figure 2 and figure 3

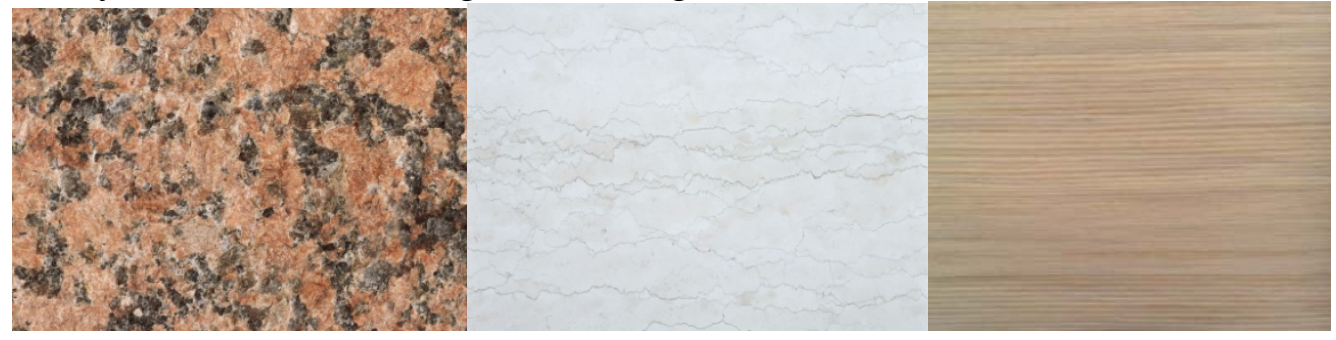

Fig 2. Natural color

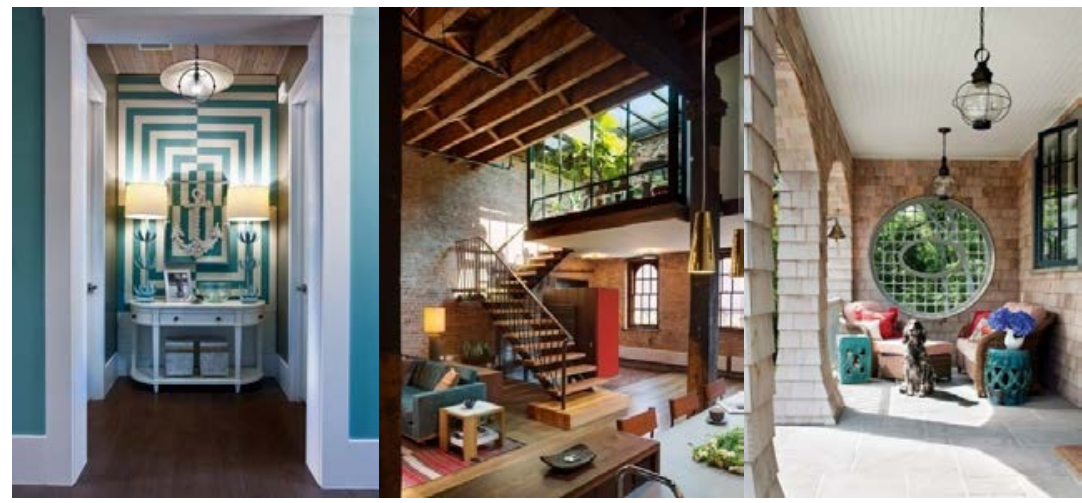

Fig 3. Secondary color

The Specifications and Performance of the Material. Material specification is refers to the shape, size, type, thickness, different specifications of the materials have different forms of aesthetic feeling, even if the same kind of material with different specifications in the form of combination, also formed different visual expression. Material specifications reflect a certain modulus, the material specification grasp can be fully the material of the decorative and practical, Material performance mainly refers to the quality of the weight, strength of soft hard, sound insulation, heat insulation, moisture, and mechanical properties, processing, wear resistance, etc.. For example, in recent years, the development of coated glass, insulating glass, laminated glass, heat reflective glass, not only 
regulate the indoor light is also with the indoor air conditioning, saving energy. All kinds of acoustic board, sound absorption coating, not only decoration the interior, but also reduce the noise. And to strengthen the fire protection of modern architecture, commonly used decorative wallpaper. Now, most of them have the antistatic, anti pollution, fire alarm, X-ray, anti moth eaten, deodorant, heat insulation and other different functions. If we do not understand the material, it may cause improper use of materials, so that not only cannot reach the effect of the decoration, and did not achieve the purpose of using

Material Process. Material process in general can be divided into early processing technology and late in the construction process, is the space and details of building design. Processing technology mainly is the attribute of material itself (such as mirror stainless steel, brushed stainless steel, scratch the glass, mirror glass, glass cracks, etc.) as well as from the designer is to obtain visual sublimation, did Such as polishing, edge grinding, concave, slot, convergent, the choice of bearing ratio, to determine the size of the details. "Cover of the bedroom, your essence is inexpensive beautiful..." Is the best judge for the process,, In fact, regardless of the east and west, architectural details since the ancient times is the place of talent, The pillar of a mountain in ancient Greece, the Assyrian glazed brick veneer, gothic period rose window and Chinese ancient wooden buildings in the so-called "mountain section algae comb", "Dan jacaranda carved Jue" and so on all surprise. Interior design is for people to build all kinds of life style, so in particular emphasis on detail design and processing. To detail design, can implement to more human physiological and psychological level, can better forms of aesthetic feeling.

\section{The Use of Materials}

"Materials not guilty, guilty is the designer", the material itself because someone attunement just makes it has a life, has the aesthetic value and different aesthetic values of the same material will produce different spatial character This shows that the use of materials in the one hand reflects the designer's quality and level.

Meets the Design Function. According to the types and functions of the space, such as hotels, hospitals, office buildings and specific to the lobby, dining room, library, bathroom, toilet, and other, in the design of material choice has a different requirements, material selection should have a more accurate positioning. Such as: hotel and the construction of the hotel is a four-star five-star level, to show the different degree of internal space of showily atmosphere, with its decorative materials. In theatres, conference room space, the need to adopt acoustic decoration materials and building technique, such as perforated plasterboard, soft fiber board, decorative acoustic board, etc. And in the bedroom space will choose environmental protection, natural, comfortable material, etc.; Decoration parts of different, the materials choice is also different, for example, we rarely see the ground in the lobby of the hotel to see the carpet, more difficult to see in the kitchen and bathroom carpet or wood floors. For small space should be chosen the delicate texture and smaller scale or expand the effect of material, and the open space, you can the deep tones and the larger pattern, reduce the space of the cold and hard.

Conforms to the Spiritual Aesthetic Needs. Material is not only satisfying the purpose of the function, but also the important subject of the interior designer. Different materials of different aesthetic feeling, just as the famous Swiss architects Herzog says "something unattractive but affects People's Daily lives, Living in a house built of concrete and live in with wood, stone house is different, the material is not only the surface of the surround close space formation, but also carry and express the thought of the house.

\section{The Unified Principle of Interior Design true Goodness and Beauty}

The ancients have "all things have spirit" the concept of " nature and humanity", reflects the concept of people's material and a kind of worship and awe of nature, while respecting the values of life. The first tool materials in human creation had already known use of stone, bronze, iron, Material 
is a kind of people and objects reflected in the relationship. Material in interior space is the most closely contact with human body, the reasonable use of materials, research it is important for people's aesthetic and psychological needs, most can show the concern's for people. Material as the carrier of emotion in the design of the pursuit of "truth, kindness and beauty", is widely accepted standards and sentiment.

Be good, one is the requirement of stylist moral quality. Design is a process of problem solving, and ultimately to serve, designspace is supposed to be a white space, give a person care and security, and meet the needs of people's physical and mental, We design the ultimate end-result is the user, this requires the designer to uphold the people-oriented design concept; The second is the pursuit material of the application , economic, long, fire prevention, strong resistance, and environmental protection.

Be beauty, "and for us. Do not lack beauty, but found that", Everything moved by human, can satisfy the function using the material can be used as material. On earth, mountains, rivers, trees and flowers, insects, fish, birds and animals and even human different race, culture, customs and way of life caused by the village, rural, urban, building, etc., are we extract material source. We won't because of the progress of science and technology, the traditional buildings such as jiangnan dwellings, caves in loess plateau, tropical rain forest of tall bamboo building, grassland and desert tents, etc. Good artistic designer is won't make rigid thinking, blind pursuit of high grade and price discrimination, the life of the material is given, a good designer is a ordinary material into the ability of aesthetic pleasure. The principle of truth can be understood as material naturally express their true colors and textures, respect the material structure and logic structure and in the shape of its real performance.

\section{Conclusion}

Material is a big topic, is a science, is a kind of style, is a kind of language, but also a consciousness, a process of practice. The use of material is the embodiment of the space concept, material memory can even imagine, achieve resonance, and feel the aesthetic pleasure. For the material, we want the greatest play to its own attributes, Material itself is sending out the simple beauty, keep the material nature, keep the value of old material, conform to the characteristics of material itself, wake up the expressive force and suitable to the characteristics of new possibilities, let the material also on the life continues, this is a mission of contemporary designers.

\section{Acknowledgment}

This paper is supported by the "science and technology research projects of Colleges in Hebei Province: Z2014007”, I express my gratitude to it.

\section{References}

[1] Q.Zhang, the research and application of modeling art materials in interior design "art and design (Theory)" in 10 (2009)

[2] Y.B. Gu; important part of interior design -- design concept and Realization; Journal of Inner Mongolia Normal University (Philosophy and Social Sciences); 03 (2004)

[3] S.R. Liu; S.Xu; the aesthetic attribute of interior decoration materials; Journal of Chifeng University (Natural Science); 07 (2009)

[4] H. Xiao; from tradition to modern; Nanjing Forestry University; (2007) 